\title{
Auto-Tracking System for Human Lumbar Motion Analysis
}

Fuge Sui ${ }^{1}$,Da Zhang ${ }^{3}$,Shing Chun Benny Lam ${ }^{4}$, Lifeng Zhao ${ }^{1}$ Dongjun Wang ${ }^{1}$,Zhenggang Bi $^{5}$,Yong $\mathrm{Hu}^{2} *$

1. Department. of Orthopaedic Surgery, Longnan Hospital, Daqing, Helongjiang, PR China

2. Department of Orthopaedics and Traumatology, The University of Hong Kong

3. Daqing Youtian Gerneral Hospital aqing, Helongjiang, PR China

4. Department of Radiology, University of Pennsylvania, Philadelphia, PA, USA

5. Department of Orthopaedic surgery, The First Affiliated Hospital of Harbin Medical University, Harbin , China

* Correspondence to: Dr. Y. Hu

12 Sandy Bay Road, Hong Kong

Duchess of Kent Children's Hospital

Department of Orthopaedics Surgery,

The University of Hong Kong

Pokfulam, Hong Kong

Tel: (852) 29740359

Fax: (852) 29740335

E-mail: yhud@hkusua.hku.hk 
Abstract

Previous lumbar motion analyses suggest the usefulness of quantitatively characterizing spine motion. However, the application of such measurements is still limited by the lack of user-friendly automatic spine motion analysis systems. This paper describes an automatic analysis system to measure lumbar spine disorders that consists of a spine motion guidance device, an X-ray imaging modality to acquire digitized video fluoroscopy (DVF) sequences and an automated tracking module with a graphical user interface (GUI). DVF sequences of the lumbar spine are recorded during flexion-extension under a guidance device. The automatic tracking software utilizing a particle filter locates the vertebra-of-interest in every frame of the sequence, and the tracking result is displayed on the GUI. Kinematic parameters are also extracted from the tracking results for motion analysis. We observed that, in a bone model test, the maximum fiducial error was 3.7\%, and the maximum repeatability error in translation and rotation was $1.2 \%$ and $2.6 \%$, respectively. In our simulated DVF sequence study, the automatic tracking was not successful when the noise intensity was greater than 0.50 . In a noisy situation, the maximal difference was $1.3 \mathrm{~mm}$ in translation and $1^{\circ}$ in the rotation angle. The errors were calculated in translation (fiducial error: $2.4 \%$, repeatability error: $0.5 \%$ ) and in the rotation angle (fiducial error: $1.0 \%$, repeatability error: $0.7 \%$ ). However, the automatic tracking software could successfully track simulated sequences contaminated by noise at a density $\leq 0.5$ with very high accuracy, providing good reliability and robustness. A clinical trial with 10 healthy subjects and 2 lumbar spondylolisthesis patients were enrolled in this study. The measurement with auto-tacking of DVF provided some information not seen in the conventional X-ray. The results proposed the potential use of the proposed system for clinical applications.

Index Terms-Auto-tracking; digitized video fluoroscopy; Spine Motion; Particle Filter; Lumbar Spine; Vertebral Body. 
2 Lumbar spine instability is an ill-defined clinical entity and is most likely related to the large 3 number of patients with chronic low back pain. Current definitions of spinal instability are 4 based on "a loss of stiffness" [1,2]. Thus, in an unstable condition, a small load results in a 5 large displacement. There has been difficulty in translating this definition into criteria that can 6 be applied to clinical diagnosis and consequent choice of treatment [3]. Clinically, physical 7 signs, such as a visible slip, catch, click or shaking of the section during motion, are commonly used for diagnosing spinal instability $[1,3,5]$.

The diagnosis of lumbar instability commonly depends on the chief complaints by the patient and plain X-ray radiography in two dimensions [4]. Radiographs taken at several different positions, such as in full extension and full flexion, are a definite and convenient way to obtain information about spine motion, but do not reflect the continuous vertebral process.

Due to the application and expansion of medical technology [5-8], digitalized video fluoroscopy (DVF) sequences [9-15] have been recommended for kinematic data acquisition of spine motion. In 1989, DVF was first applied to investigate spine kinematics by Breen et al. [14]. The advantages include a low level of intervention, a low-dose X-ray and continuous imaging for moving vertebrae. This special imaging technology laid the groundwork for recording spine motion in vivo. Many biomedical engineering studies [10, 13, 14, 16-27] have analyzed spine biomechanics and have presented methods to identify and mark vertebral corners, as well as tracking algorithms for vertebrae. However, relevant information regarding realization and application at clinical system levels is rarely reported. Therefore, we combined all of the necessary components for a comprehensive system, denoted as the Vertebrae Analysis System (VAS), which allows the study of lumbar vertebrae movement in models and in vivo. The results from the VAS provide an objective basis for lumbar disorder diagnoses.

The VAS is capable of tracking the vertebrae of the lumbar spine and to estimate the dynamic motion of the spine in most cases. Breen et al. [14], who firstly introduced DVF to investigate 
1 spine kinematics, succeeded in using DVF to acquire and analyze lumbar spine motion.

2 Kondracki consolidated the usefulness of DVF in spine motion with a passive motion table

3 [28]. Okawa et al. [11] used a sandwich stand to assist in video fluoroscopy acquisition from

4 subjects with and without back pain. Teyhen et al. [13] proposed methods for video

5 fluoroscopy image enhancement and distortion-compensated roentgen analysis, as well as

6 showed the reliability of their methods and demonstrated an improvement in video

7 fluoroscopy image measurements. However, the main limitation of their study was that the

8 vertebral motions could only be recorded at certain fixed frames or time intervals. Lee et al.

9 [9] and Wong et al. [28] evaluated the inter-vertebral motion at certain fixed anatomic ranges

10 of lumbar spine motion, which was not a time-dependent parameter. Notably, the VAS

11 overcomes these limitations. The development of auto-tracking technology was mainly

12 attributed to the application of DVF and the improvement of tracking algorithms [18].

13 In this study, an attempt was made to gain the motion trajectories of model lumbar vertebrae

14 to test the robustness and the reliability of the VAS. Specifically, clinical practicability was

15 illustrated by importing a healthy human DVF sequence into the auto-tracking system. 
1

2

3 Each DVF sequence was collected using Philips Digital Subtraction Angiography (Philips

4 Medical Systems, Netherlands). The system was in a horizontal position as shown in Fig. 1.

5 DVF sequences were stored and processed on a desk-top computer.

6 An automatic guidance device was custom-designed to keep the subject (model or human)

7 aligned, in order to minimize out-of-plane motion during constant speed sagittal flexion-

\section{Materials and methods}

2.1. Instrumentation extension, as shown in Fig. 1 (a). The guide device included a control unit, an air cylinder and a rising and dropping stool. The subject sat on the stool, and their hip was held against the stool with a seat belt in order to keep the sacral vertebrae from sliding (Fig. 1 (b)).

\subsection{Image preprocessing}

Each DVF image sequence had a low signal to noise ratio due to the low X-ray dose imaging mode employed in our setup, while the contrast between vertebrae and surrounding tissue was degraded. In order to enhance the image quality and to facilitate automated tracking, twodimensional median filtering was applied in histogram equalization to the images. This application was employed to preserve a clear vertebra boundary and to minimize noise as much as possible.

The executable software used in the VAS was developed for both automatic tracking and optional manual locating algorithms. The operation window was developed with the open Graphical User Interface (GUI) (Fig 2).

Landmarking the vertebrae in a DVF sequence, which can be done automatically, is the basis of kinematic analysis. The previous publication claimed that automatic vertebra tracking may not be succeed in a very poor imaging [24]. Therefore, this system remain a function to allow the operator to manually place the markers on the four vertebra corners (two dorsal corners and two ventral corners) in each vertebra of the start frame of the DVF sequence. Trajectory 
analysis was performed by local polynomial regression analysis [29] to construct a smooth curve graph, after which the translation and angle of rotation of the vertebrae were obtained.

\subsection{Automated tracking module}

The proposed VAS adopted the automated vertebra tracking algorithm reported in [23]. The tracking algorithm utilizes particle filter to estimates the posterior distribution of the $\mathrm{x}$ - and $\mathrm{y}$ displacement $\left(\Delta x_{t}, \Delta y_{t}\right)$ and the change in orientation $\left(\Delta \theta_{t}\right)$ of the vertebra from frame $t$ to $t+1$, which are formulated in a state vector as

$$
\mathrm{X}_{t}=\left[\Delta x_{t}, \Delta y_{t}, \Delta \theta_{t}\right]^{\mathrm{T}}
$$

The particle filter estimates the posterior distribution $p\left(\mathrm{X}_{t} \mid \mathrm{Z}_{1: t}\right)$ of $\mathrm{X}_{t}$ from a noisy collection of observations (or measurements) $Z_{1: t}=\left(Z_{1}, Z_{2}, \cdots, Z_{t}\right)$ from each frame of the fluoroscopic sequence arriving in a sequential fashion. From frame $t-1$ to $t$, the particle filter generates $N$ samples (called particles) according to the prior distribution of a state transition model $p\left(\mathrm{X}_{t} \mid \mathrm{X}_{t-1}\right)$ for each vertebra to predict the location and orientation in the frame $t$. The observation model measures the goodness of fit between the projected spline contour (according to the particles) and the vertebral edge. This forms the likelihood distribution $p\left(Z_{t} \mid X_{t}\right)$ of the measurements. Here, the scoring function of measurement of contour, $\varphi_{t}$, is formulated as the sum of gradient magnitude squared under the spline contour $A\left(C_{t}\right)$, where $\mathrm{C}_{\mathrm{t}}$ is a vector contains all the control points that constitute the contour. Let the contour giving the highest score be $\max \left(\varphi_{t}\right)$, the likelihood distribution

$$
p\left(Z_{t} \mid X_{t}^{(n)}\right) \mid \propto \exp \left(\frac{\left|\max \left(\varphi_{t}\right)-\varphi_{t}^{(n)}\right|}{2}\right)
$$

where $\mathrm{n}$ denotes the number of particles.

By applying Sequential Importance Resampling (SIR) to each time step to prevent degeneracy problems [30], the weight for each particle $n$ becomes

$$
\omega_{t}^{(n)} \propto p\left(Z_{t} \mid X_{t}^{(n)}\right) \quad(3) \text { and }
$$




$$
p\left(X_{t} \mid Z_{1: t}\right) \approx \sum_{n=1}^{N} \omega_{t}^{(n)} \delta\left(X_{t}-X_{t}^{(n)}\right)
$$

where $\delta(\bullet)$ is a Dirac delta function. The kinematic parameters in the state vector $\hat{\mathbf{X}}_{t}$ at frame $t$ were computed using the maximum likelihood (ML) estimate

$$
\hat{X}_{t} \approx \frac{1}{N} \sum_{n=1}^{N} \omega_{t}^{(n)} X_{t}^{(n)}
$$

after which $\mathrm{C}_{t}$ is updated with $\hat{\mathbf{X}}_{t}$ and return to the particle filter for the next iteration.

\subsection{Performance assessment with simulation studies and human subjects}

To assess the robustness and the reliability of the VAS, a simulation study using a bone model was performed. The DVF of the bone model was obtained at $45 \mathrm{kV}, 80 \mathrm{~mA}$ and an exposure time of $3 \mathrm{~ms}$. During the DVF acquisition process, the L1 to L5 region was maintained within the field of view. The assisting device also pulled and pushed the plastic model of the lumbar vertebral column (Anatomical lambo-sacral model, Ortholink LLC, CA, USA) to perform sagittal cycling flexion-extension motion. The continuous dynamic lumbar sequence of the model was assessed in four cycles. When the collection was finished, each vertebra trajectory was recorded by a real-time depiction of the vertebral body with a fixedpen and chart paper recorder and was used for a benchmark of the vertebral motion analysis. The measurement accuracy in terms of the $\mathrm{x}$-, $\mathrm{y}$-translations and rotation angles was calculated in comparison with the benchmark. Additionally, the reproducibility was evaluated by measurement errors in the cycles.

To test the robustness of the VAS to noise, a new sequence was produced by adding noise with differing densities to degrade the image quality. The simulated sequence consisted of 61 frames of a drawn vertebra in motion. The vertebra moved by $1 \mathrm{~mm}$ in $\mathrm{x}$ - and $\mathrm{y}$-translations and by $1^{\circ}$ in the rotation angle in each frame of the simulated sequence. The range of motion was preset respectively at -20 to $10 \mathrm{~mm}$ and 20 to $-10 \mathrm{~mm}$ in the $\mathrm{x}$ - and y-translations and $20^{\circ}$ 
1 to $-10^{\circ}$ in the rotation angle by $1 \mathrm{~mm}$ and $1^{\circ}$ between the adjacent frames. The histogram

2 equalization and filter were not applied to the specific sequence. The intensity values of the

3 sequence were scaled to [0 1], such that both the image and noise were of the same scale.

4 To evaluate the clinical usefulness of the VAS, 10 healthy subjects and 2 lumbar degenerated 5 patients were enrolled in the study with informed consent. There were 3 female and 7 male 6 healthy subjects, aged from 19 to 25 at mean of 23.5 years old, without any evidence of 7 lumbar degenerative disease. One female patient at 38 years old was diagnosed with L5/S1 8 lumbar spondylolisthesis, confirmed with radiological detection. Another male patient at 38 9 years old was also diagnosed as L5/S1 lumbar spondylolisthesis without obvious radiological evidence. The DVF sequences of the healthy subjects and patients were obtained by the same medical system $(68 \mathrm{kV}, 300 \mathrm{~mA}$, exposure time: $7 \mathrm{~ms})$. Each subject was asked to sit in their neutral position and led by the guide device to perform sagittal flexion-extension motion

13 starting from this position. The L1 to S region of the lumbar was maintained within the field of view while the DVF sequence was collected. Image sequences were taken at a lateral projection. The normal parameters of $\mathrm{x}$-translation and the rotation angle were calculated in the control group from the extension to flexion position with time normalization. The $\mathrm{x}$ translation and rotation angle in the patients were measured and then compared with the data from the healthy subjects. 


\section{Results}

3.1. Reliability assessment with an anatomical lambo-sacral model

A bone model from L1 to the sacrum was used for the assessment of the VAS. Figure 3 partly plots frames from the DVF sequence of the tracking process. The trajectories of the lumbar vertebrae model (L1-L5) motion were recorded as benchmarks. In the 4 integral cycles, the fiducial errors of $\mathrm{x}$ - and $\mathrm{y}$-translations were calculated correspondingly between the automatic tracking and actual measurements (Table 1). The maximum of the fiducial error was $3.7 \%$ in $x$-translation. The root mean square differences (RMS) and the standard error of the measurement (SEM) [31] of the rotation angle, as well as the $\mathrm{x}$ - and $\mathrm{y}$-translations, were calculated to test the variability and robustness of the VAS. The average RMS differences of the x-translation, y-translation and angle of rotation were 0.69 (SD 0.4) mm, 0.64 (SD 0.3) $\mathrm{mm}$ and $0.9^{\circ}\left(\mathrm{SD} 0.3^{\circ}\right)$, while the SEM was $0.47,0.42$ and $0.57^{\circ}$, respectively, as shown in Table 2. The mean and standard deviation of the intraclass correlation coefficient [31] (ICC) is shown in Table 3. The average ICCs of the $\mathrm{x}$ - and y-translations were 0.99 (SD 0.009) and 0.99 (SD 0.005), respectively, between the auto-tracking and actual measurements.

\subsection{Robustness assessment with a simulated sequence}

A simulated sequence was measured in order to assess the performance of the auto-tracking system during measurements of low quality imaging. The densities of added noise were 0.10 , $0.30,0.50$ and 0.70 . The example of an image contaminated by "salt \& pepper" is shown in Fig. 4. The tracking process failed with a noise density of 0.70 . Therefore, subsequent analyses included simulations at noise densities of $0.10,0.30$ and 0.50 . For auto tracking in the VAS, each sequence was initialized by placing approximately 50 control points along the vertebral edges on the first frame. Each vertebra used 2000 particles, and the original point of $\left[\Delta \mathrm{x}_{\mathrm{t}}^{2}, \Delta \mathrm{y}_{\mathrm{t}}^{2}, \Delta \theta_{\mathrm{t}}^{2}\right]$ was set as $[4,1,1]$.

Simulated results, representing the average value of 6 trials, showed a fiducial error of $2.4 \%$ in $\mathrm{x}$-translation, $2.4 \%$ in y-translation and $1.0 \%$ in rotation angle (Table 4). The repeatability 
error was $0.5 \%, 0.5 \%$, and $0.7 \%$, respectively, in the $\mathrm{x}$ - and $\mathrm{y}$-translations and in the rotation angle. In the sequence with the added "salt \& pepper" noise (density $=0.10,0.30$ and 0.50 ), the tracking results were good and did not show obvious differences with the increasing noise densities.

\subsection{Performance in human subjects}

Using the guidance system, the scope of lumbar flexion and extension was acquired from each subject. The DVF sequences were then analyzed by the VAS system. For the analysis of the DVF model sequences, each vertebra used 2000 particles, and the original point of $\left[\Delta \mathrm{x}_{\mathrm{t}}^{2}, \Delta \mathrm{y}_{\mathrm{t}}^{2}, \Delta \theta_{\mathrm{t}}^{2}\right]$ was set as $[4,1,1]$.

Each subject was asked to perform a complete flexion-extension cycle, to/from straight sitting to extension/flexion (Figure 5). Healthy subjects showed a rotation angle pattern in a complete flexion-extension cycle as depicted in Figure 6, where the blue solid line presents the mean value of 10 healthy subjects, and the green and light-blue dotted lines present the range $( \pm 2.5 \mathrm{SD})$ of normal values. The trace of vertebra angles during the flexion-extension of a 38-years-old female, who was diagnosed with L5 spondylolisthesis with obvious radiography disability in the L5-S disc level (Figure 7(a)), is plotted in Figure 6(a), demonstrating that the VAS results confirmed the radiographical diagnosis. In case 2, a 53year-old female presented with symptoms of spondylolisthesis without obvious radiology presentation during standing, flexion and extension X-ray examination (Figure 7(b)). However, the DVF analysis indicated L5 spondylolisthesis during dynamic movement, as shown in Fig 6(b). The results of the rotation angle in Figure 6(b) indicate abnormal rotation in a short period of flexion-extension, which was not identified by traditional X-ray in standing, flexion or extension postures. 


\section{Discussion}

The VAS was developed into a medical system for clinical application. It is capable of tracking the vertebrae of the lumbar spine and to estimate the dynamic motion of the spine in most cases. The development of auto-tracking technology was mainly attributed to the application of DVF and the improvement of tracking algorithms [18]. Breen et al. [14], who firstly introduced DVF to investigate spine kinematics, succeeded in using DVF to acquire and analyze lumbar spine motion. Kondracki consolidated the usefulness of DVF in spine motion with a passive motion table [28]. Okawa et al. [11] used a sandwich stand to assist in video fluoroscopy acquisition from subjects with and without back pain. Teyhen et al. [13] proposed methods for video fluoroscopy image enhancement and distortion-compensated roentgen analysis, as well as showed the reliability of their methods and demonstrated an improvement in video fluoroscopy image measurements. However, the main limitation of their study was that the vertebral motions could only be recorded at certain fixed frames or time intervals. Lee et al. [9] and Wong et al. [28] evaluated the inter-vertebral motion at certain fixed anatomic ranges of lumbar spine motion, which was not a time-dependent parameter. Notably, the VAS overcomes these limitations. With its clinical application, it is possible to standardize the spine motion and to quantify the pattern of spinal movement.

In this study, the radiography system selected the minimal X-ray exposure automatically (based on a setting threshold of $45 \mathrm{kV}, 80 \mathrm{~mA}$ and an exposure time of $3 \mathrm{~ms}$ ). The results were compared with a standard radiological dose of $68 \mathrm{kV}, 300 \mathrm{~mA}$ and an exposure time of $7 \mathrm{~ms}$. The DVF sequences under the lower dose were tracked completely, as well as those obtained under the higher dose. This result indicates that the VAS performed robustly when the dose was higher than or equal to the lower radiological dose.

The measurement model of the particle filter employed in this study considers the nature of image formation of X-ray DVF [18]. A simulation study was performed which compared the RMS error between the particle filter-estimated angle of rotation and translation (i.e. tracking results) with corresponding preset values. We found that a particle filter of the same type 
1 achieved an RMS error for less than 0.2 degrees and 0.5 pixels in the angle of rotation and

2 translation, respectively, when the Gaussian noise of the normalized variance $(\sigma 2)$ was less

3 than 0.65 . The particle filter-tracker slightly tolerated speckled-noise, whereas it failed when

$4 \sigma 2>0.75$. These results suggest that our particle filter exhibited a significantly high tolerance

5 to different densities of noise.

6 In the present bone model and simulation study, the automatic tracking results were very

7 close to the actual measurements or the preset values. The translation and angle were accurate

8 in the limited flexion-extension range. In the human subjects, the use of a guidance system

9 provided an efficient examination, without any out-of-plane effects. When the developed

10 VAS system was applied to the lumbar degenerated patients, an accurate diagnosis was made

11 for both patients, in particular for one patient when the traditional X-ray did not show an

12 abnormal pattern. These preliminary results suggest the potential use of this new system for

13 the clinical diagnosis of spondylolisthesis.

14 Notably, the small sample size (10 healthy subjects and 2 symptom-presenting patients) is a 15 limiting factor in this study, and we require additional subjects with and without lumbar spine 16 problems for further comparisons. A large scale prospective randomized double blind clinical 17 trial should be investigated to evaluate the clinical usefulness of this in vivo diagnosis of 18 lumbar disorders. 


\section{5. Conclusions}

2 The proposed VAS provides a tool to investigate lumbar disorders. The automated tracking 3 module performed with significant robustness and reliability in tracking the motions from

4 DVF sequences. Our proposed VAS was evaluated by bone models, simulated sequences and 5 human subjects. Collectively, we found that the auto-tracking algorithm produced results with 6 acceptable accuracy, good reliability and robustness, suggesting that the proposed VAS 7 should be considered for further clinical trial evaluations.

\section{Acknowledgment}

10 This work was supported in part by Institute of Biomedical Engineering of Perking Union 11 Medical College \& Chinese Academy of Medical Sciences and Daqing Petroleum 12 Administrative Bureau. The authors thank Dr Xueming Wang for assistance in the process of 13 tailor-made guide device and Miss Anna Lee for the language editing. 


\section{References}

[1] P. M. White A, "Kinematics of the spine," Clinical Biomechanics of the Spine P. M. White A, ed., Philadelphia: J.B. Lippincott, 1990.

[2] M. H. Pope, and M. Panjabi, "Biomechanical definitions of spinal instability," Spine (Phila Pa 1976), vol. 10, no. 3, pp. 255-6, Apr, 1985.

[3] F. J., P. M., and W. D., “ Segmental instability. In The Lumbar Spine Edited by Wiesel S, Weinstein J, Herkowitz H, et al. 2nd Ed. W. B. Saunders, Philadelphia. ," 1996.

[4] F. Ringel, M. Stoffel, C. Stuer et al., "Minimally invasive transmuscular pedicle screw fixation of the thoracic and lumbar spine," Neurosurgery, vol. 59, no. 4 Suppl 2, pp. ONS3616; discussion ONS366-7, Oct, 2006.

[5] J. M. Olsewski, E. H. Simmons, F. C. Kallen et al., "Morphometry of the lumbar spine: anatomical perspectives related to transpedicular fixation," Journal of Bone and Joint Surgery-American Volume, vol. 72, no. 4, pp. 541-9, Apr, 1990.

[6] M. M. Panjabi, V. Goel, T. Oxland et al., "Human lumbar vertebrae. Quantitative threedimensional anatomy," Spine (Phila Pa 1976), vol. 17, no. 3, pp. 299-306, Mar, 1992.

[7] M. M. Panjabi, K. Takata, V. Goel et al., "Thoracic human vertebrae. Quantitative threedimensional anatomy," Spine (Phila Pa 1976), vol. 16, no. 8, pp. 888-901, Aug, 1991.

[8] A. White, and P. M, "Kinematics of the spine," Clinical Biomechanics of the Spine P. M. White A, ed., Philadelphia: J.B. Lippincott, 1990.

[9] S. W. Lee, K. W. Wong, M. K. Chan et al., "Development and validation of a new technique for assessing lumbar spine motion," Spine (Phila Pa 1976), vol. 27, no. 8, pp. E215-20, Apr $15,2002$.

[10] A. Breen, R. Allen, and A. Morris, "A digital videofluoroscopic technique for spine kinematics,” J Med Eng Technol, vol. 13, no. 1-2, pp. 109-13, Jan-Apr, 1989.

[11] A. Okawa, K. Shinomiya, H. Komori et al., "Dynamic motion study of the whole lumbar spine by videofluoroscopy," Spine (Phila Pa 1976), vol. 23, no. 16, pp. 1743-9, Aug 15, 1998.

[12] D. Vander Kooi, G. Abad, J. R. Basford et al., "Lumbar spine stabilization with a thoracolumbosacral orthosis: evaluation with video fluoroscopy," Spine (Phila Pa 1976), vol. 29, no. 1, pp. 100-4, Jan 1, 2004.

[13] D. S. Teyhen, T. W. Flynn, A. C. Bovik et al., "A new technique for digital fluoroscopic video assessment of sagittal plane lumbar spine motion," Spine (Phila Pa 1976), vol. 30, no. 14, pp. E406-13, Jul 15, 2005.

[14] A. C. Breen, R. Allen, and A. Morris, "Spine kinematics: a digital videofluoroscopic technique," J Biomed Eng, vol. 11, no. 3, pp. 224-8, May, 1989.

[15] K. Takayanagi, K. Takahashi, M. Yamagata et al., "Using cineradiography for continuous dynamic-motion analysis of the lumbar spine," Spine, vol. 26, no. 17, pp. 1858-1865, Sep 1, 2001.

[16] M. Panjabi, D. Chang, and J. Dvorak, "An analysis of errors in kinematic parameters associated with in vivo functional radiographs," Spine (Phila Pa 1976), vol. 17, no. 2, pp. 200-5, Feb, 1992. 
[17] Y. L. Zheng, M. S. Nixon, and R. Allen, "Automated segmentation of lumbar vertebrae in digital videofluoroscopic images," Ieee Transactions on Medical Imaging, vol. 23, no. 1, pp. 45-52, Jan, 2004.

[18] S. C. B. Lam, B. McCane, and R. Allen, "Automated tracking in digitized videofluoroscopy sequences for spine kinematic analysis," Image and Vision Computing, vol. 27, no. 10, pp. 1555-1571, Sep 2, 2009.

[19] P. Bifulco., M. Cesarelli., R. Allen. et al., "Automatic extraction of kinematic parameters from videofluoroscopic sequences of the spine," Health Telematics, Naples, Italy, pp. 147$148,1995$.

[20] J. M. Muggleton, and R. Allen, "Automatic location of vertebrae in digitized videofluoroscopic images of the lumbar spine," Medical Engineering \& Physics, vol. 19, no. 1, pp. 77-89, Jan, 1997.

[21] A. Thorkeldsen, and A. C. Breen, "Gray scale range and the marking of vertebral coordinates on digitized radiographic images," J Manipulative Physiol Ther, vol. 17, no. 6, pp. 359-63, Jul-Aug, 1994.

[22] C. Cardan., and R. Allen., "Measurement of spine motion for diagnosis of mechanical problems," J. Comp. Simu. Mod. Med, vol. 1, pp. 15-19, 2000.

[23] S. C. B. Lam, R. Allen, G. Pearson et al., "Parametric characterization of spinal motions in osteoporotic vertebral fracture at level T12 with fluoroscopy," Medical Engineering \& Physics, vol. 31, no. 3, pp. 346-355, Apr, 2009.

[24] W. Frobin, P. Brinckmann, G. Leivseth et al., "Precision measurement of segmental motion from flexion-extension radiographs of the lumbar spine," Clin Biomech (Bristol, Avon), vol. 11, no. 8, pp. 457-465, Dec, 1996.

[25] C. Simonis., R. Allen., and a. R. Cloke, "Spatial analysis of the movement of the spine: application of parallel computing in the field of spine biomechanics," Transputer Applications, vol. 1, pp. 35-38, 1993.

[26] P. P. Smyth, C. J. Taylor, and J. E. Adams, "Vertebral shape: automatic measurement with active shape models," Radiology, vol. 211, no. 2, pp. 571-8, May, 1999.

[27] M. G. Roberts, T. F. Cootes, and J. E. Adams, "Vertebral shape: Automatic measurement with dynamically sequenced active appearance models," Medical Image Computing and Computer-Assisted Intervention - Miccai 2005, Pt 2, vol. 3750, pp. 733-740, 2005.

[28] K. W. Wong, J. C. Leong, M. K. Chan et al., "The flexion-extension profile of lumbar spine in 100 healthy volunteers," Spine (Phila Pa 1976), vol. 29, no. 15, pp. 1636-41, Aug 1, 2004.

[29] Z. G. Zhang, S. C. Chan, K. L. Ho et al., "On bandwidth selection in local polynomial regression analysis and its application to multi-resolution analysis of non-uniform data," Journal of Signal Processing Systems for Signal Image and Video Technology, vol. 52, no. 3, pp. 263-280, Sep, 2008.

[30] G. Kitagawa, "Monte carlo filter and smoother for non-gaussian non-linear state space models," Comput. Graph. Statist., vol. 5, no. 1, pp. 1-25, 1996.

[31] M. Bland, An introduction to medical statistics, 3rd ed ed., New York: Oxford University Press, 2000. 
2 The fiducial error between the tracking results and the actual measurement and the 3 repeatability error in first 4 tracking trails

4

\begin{tabular}{|c|c|c|c|}
\hline Vertebra & & Fiducial Error (\%) & $\begin{array}{c}\text { Repeatability } \\
\text { Error (\%) }\end{array}$ \\
\hline \multirow{3}{*}{ L1 } & $\mathrm{x}$ & 3.7 & 0.8 \\
\hline & y & 2.3 & 0.6 \\
\hline & $\theta$ & -- & 2.6 \\
\hline \multirow{3}{*}{ L2 } & $\mathrm{x}$ & 2.5 & 0.6 \\
\hline & y & 1.6 & 0.8 \\
\hline & $\theta$ & -- & 1.1 \\
\hline \multirow{3}{*}{ L3 } & $\mathrm{x}$ & 1.9 & 0.5 \\
\hline & y & 1.8 & 1.2 \\
\hline & $\theta$ & -- & 1.0 \\
\hline \multirow{3}{*}{ L4 } & $\mathrm{x}$ & 0.7 & 0.5 \\
\hline & y & 2.6 & 1.0 \\
\hline & $\theta$ & -- & 1.4 \\
\hline \multirow{3}{*}{ L5 } & $\mathrm{x}$ & 0.3 & 0.1 \\
\hline & y & 0.5 & 0.4 \\
\hline & $\theta$ & -- & 2.2 \\
\hline
\end{tabular}


RMS and SEM of the model tracking results

\begin{tabular}{|c|c|c|c|c|}
\hline \multirow{2}{*}{ Vertebra } & \multicolumn{4}{|c|}{ Sagittal flexion-extension } \\
\hline & & $x(\mathrm{~mm})$ & $\mathrm{y}(\mathrm{mm})$ & degree \\
\hline \multirow{3}{*}{ L1 } & RMSD & $1.13(0.53)$ & $0.63(0.28)$ & $1.019(0.11)$ \\
\hline & & & & \\
\hline & SEM & 0.75 & 0.41 & 0.62 \\
\hline \multirow{3}{*}{ L2 } & RMSD & $0.85(0.39)$ & $1.10(0.61)$ & $0.69(0.07)$ \\
\hline & & & & \\
\hline & SEM & 0.57 & 0.76 & 0.42 \\
\hline \multirow{3}{*}{ L3 } & RMSD & $0.67(0.34)$ & $0.49(0.08)$ & $0.64(0.07)$ \\
\hline & & & & \\
\hline & SEM & 0.45 & 0.30 & 0.39 \\
\hline \multirow{3}{*}{ L4 } & RMSD & $0.73(0.46)$ & $0.79(0.30)$ & $0.91(0.10)$ \\
\hline & & & & \\
\hline & SEM & 0.51 & 0.51 & 0.56 \\
\hline \multirow{3}{*}{ L5 } & RMSD & $0.09(0.01)$ & $0.18(0.02)$ & $1.31(0.56)$ \\
\hline & & & & \\
\hline & SEM & 0.05 & 0.11 & 0.86 \\
\hline \multirow{3}{*}{ Averaging } & RMSD & $0.69(0.4)$ & $0.64(0.3)$ & $0.9(0.3)$ \\
\hline & & & & \\
\hline & SEM & 0.47 & 0.42 & 0.57 \\
\hline
\end{tabular}

3

$4 \quad$ RMSD: Root mean square differences among first 4 tracking trials are presented as 5 mean (standard deviation).

SEM: Standard error of the measurement among first 4 tracking trails. 
2 The mean and standard deviation of ICC (Intraclass Correlation Coefficient) in 20 3 intergral cycles between the tracking results and the actual measurement and in the test-retest in 20 tracking trails

\begin{tabular}{|c|c|c|c|}
\hline \multicolumn{2}{|c|}{ Vertebra } & $\begin{array}{l}\text { Statistical Value of ICC } \\
\qquad(\mathbf{p}<0.05)\end{array}$ & $\begin{array}{l}\text { Statistical Value of ICC } \\
\text { ( Test-Retest, } p<0.05 \text { ) }\end{array}$ \\
\hline \multirow{3}{*}{ L1 } & $\mathrm{x}$ & $0.994 \pm 0.004$ & $0.9999 \pm 0.0001$ \\
\hline & $\mathrm{y}$ & $0.991 \pm 0.005$ & $0.9998 \pm 0.0048$ \\
\hline & $\Theta$ & -- & $0.9977 \pm 0.0016$ \\
\hline \multirow{3}{*}{ L2 } & $\mathrm{x}$ & $0.992 \pm 0.004$ & $0.9999 \pm 0.0001$ \\
\hline & $\mathrm{y}$ & $0.990 \pm 0.005$ & $0.9995 \pm 0.0002$ \\
\hline & $\Theta$ & -- & $0.9989 \pm 0.0003$ \\
\hline \multirow{3}{*}{ L3 } & $\mathrm{x}$ & $0.993 \pm 0.005$ & $0.9999 \pm 0.0001$ \\
\hline & $\mathrm{y}$ & $0.986 \pm 0.013$ & $0.9994 \pm 0.0003$ \\
\hline & $\theta$ & -- & $0.9984 \pm 0.0010$ \\
\hline \multirow{3}{*}{ L4 } & $\mathrm{x}$ & $0.992 \pm 0.006$ & $0.9997 \pm 0.0002$ \\
\hline & $\mathrm{y}$ & $0.986 \pm 0.010$ & $0.9987 \pm 0.0006$ \\
\hline & $\Theta$ & -- & $0.9981 \pm 0.0006$ \\
\hline \multirow{3}{*}{ L5 } & $\mathrm{x}$ & $0.974 \pm 0.020$ & $0.9954 \pm 0.0022$ \\
\hline & $\mathrm{y}$ & $0.978 \pm 0.014$ & $0.9936 \pm 0.0046$ \\
\hline & $\Theta$ & -- & $0.9933 \pm 0.0026$ \\
\hline
\end{tabular}




\section{Table 4}

Mean and standard of tracking results in simulated sequence (original) in 6 trials and the results of adding noise (salt \& pepper) with different density

\begin{tabular}{|c|c|c|c|c|c|c|c|c|c|c|c|c|}
\hline \multirow[t]{2}{*}{ Preset } & \multicolumn{4}{|c|}{$\mathrm{x}$-translation $(\mathrm{mm})$} & \multicolumn{4}{|c|}{$\mathrm{y}$-translation $(\mathrm{mm})$} & \multicolumn{4}{|c|}{ Rotation Angle $\left({ }^{\circ}\right)$} \\
\hline & $\overline{-5}$ & -10 & -15 & -20 & -5 & -10 & -15 & -20 & 5 & 10 & 15 & 20 \\
\hline Mean(Std) & $-5.4(0.1)$ & $-10.8(0.1)$ & $-16.3(0.1)$ & $-20.7(0.1)$ & $-5.4(0.1)$ & $-10.8(0.1)$ & $-16.3(0.1)$ & $-20.7(0.1)$ & $5.1(0.2)$ & $10.1(0.2)$ & $15.2(0.2)$ & $19.4(0.2)$ \\
\hline density(0.1) & -5.3 & -10.7 & -16.2 & -20.5 & -5.3 & -10.7 & -16.2 & -20.5 & 5.1 & 10.5 & 15.2 & 19.0 \\
\hline density(0.3) & -5.2 & -10.6 & -16.0 & -20.4 & -5.2 & -10.6 & -16 & -20.4 & 5.2 & 10.3 & 15.1 & 19.0 \\
\hline density(0.5) & -5.0 & -10.5 & -15.9 & -20.3 & -5.0 & -10.5 & -15.9 & -20.3 & 5.0 & 10.2 & 15.3 & 19.2 \\
\hline Maximum error & $2.4 \%$ & & & & $2.4 \%$ & & & & $1.0 \%$ & & & \\
\hline repeatability error & $0.5 \%$ & & & & $0.5 \%$ & & & & $0.7 \%$ & & & \\
\hline
\end{tabular}

Mean (Std): mean and standard of tracking results in simulated sequence (original or no adding noise) in 6 trials. 
Figure captions

Figure 1 The medical data acquisition system and motion

(a) Illustration of whole system. (b) The subject perform sitting flexion-extension movement under guidance.

Figure 2 Operation Interface.

Figure 3 An example of the lumbar model tracking results (sagittal bending)

Figure 4 The original and contaminated image in the simulated sequences. (a) The original image for simulating. (b) the images of the sequence are contaminated by "salt \& pepper" (density $=0.50)$.

Figure 5 Extractions of the tracking results with healthy huam DVF sequence (sagittal bending)

Figure 6 Rotation angle analysis of lumbar motion (a) Case 1 verse normal group , (b) Case 2 verse normal group.

Figure 7 X-ray examination (a) Flexion X-ray of Case 1, (b) Standing X-Ray of Case 1, (c) Extension X-ray of Case 1, (d) Flexion X-ray of Case 2, (e) Standing X-Ray of Case 2, (f) Extension X-ray of Case 2. 
Figure 1

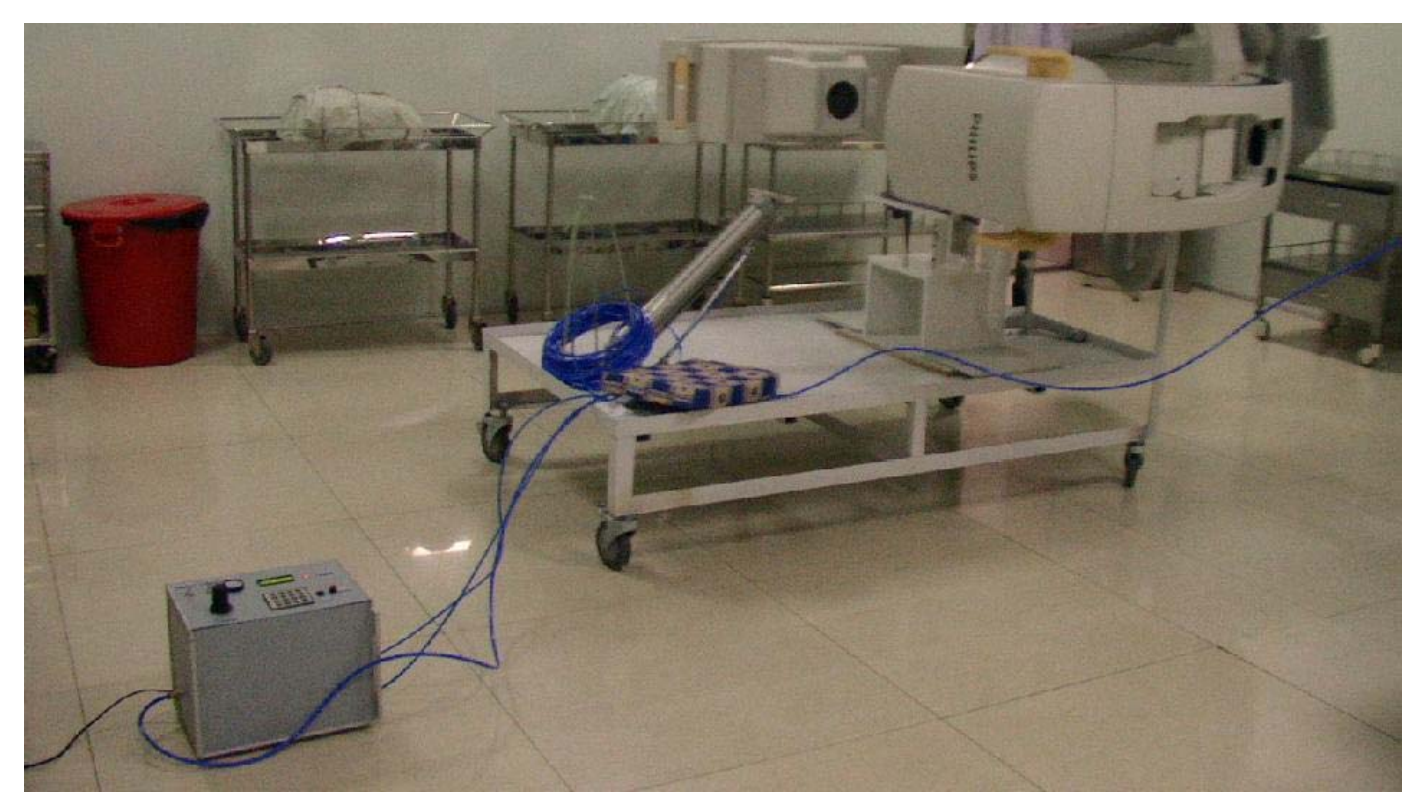

(a)

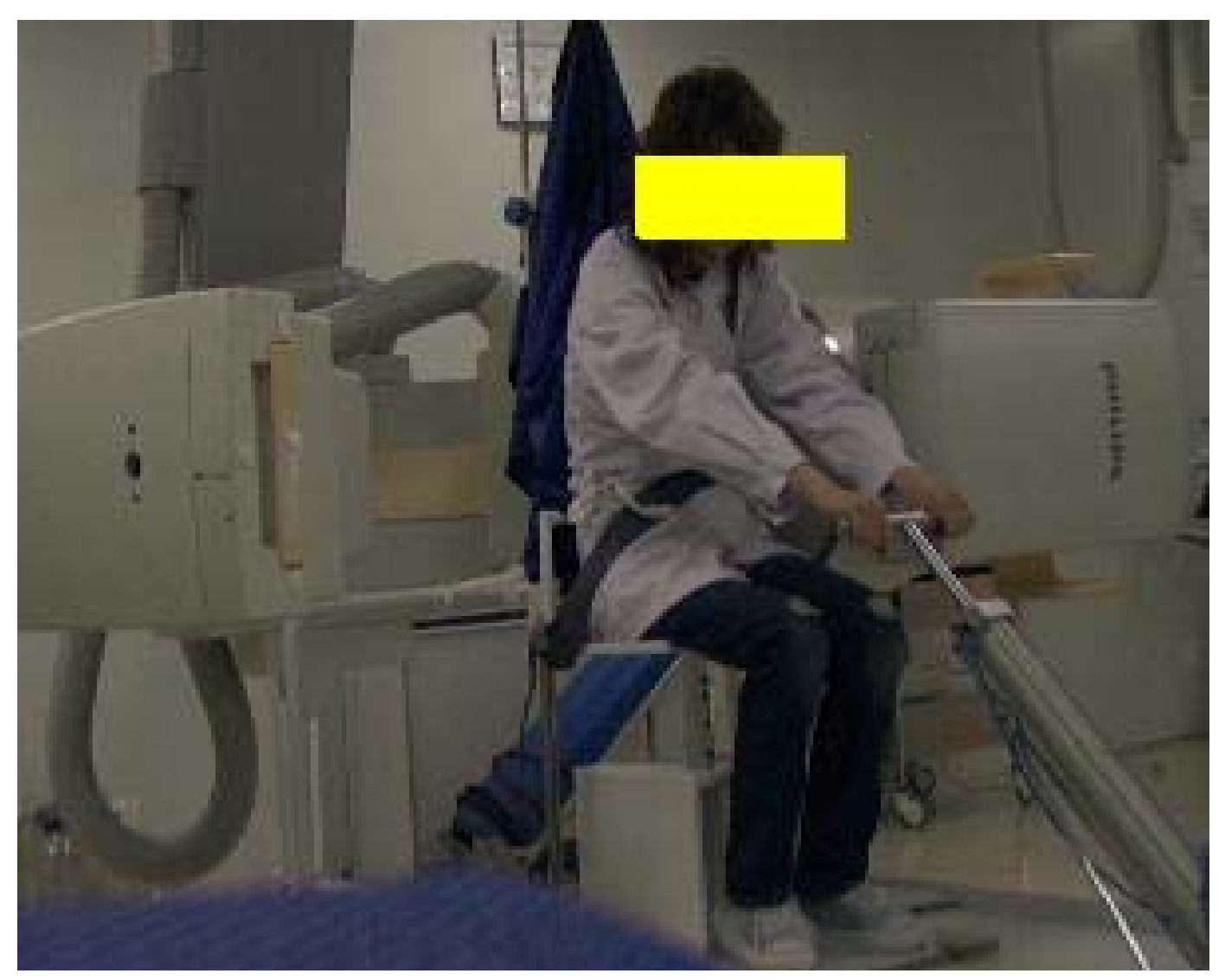


Figure 2

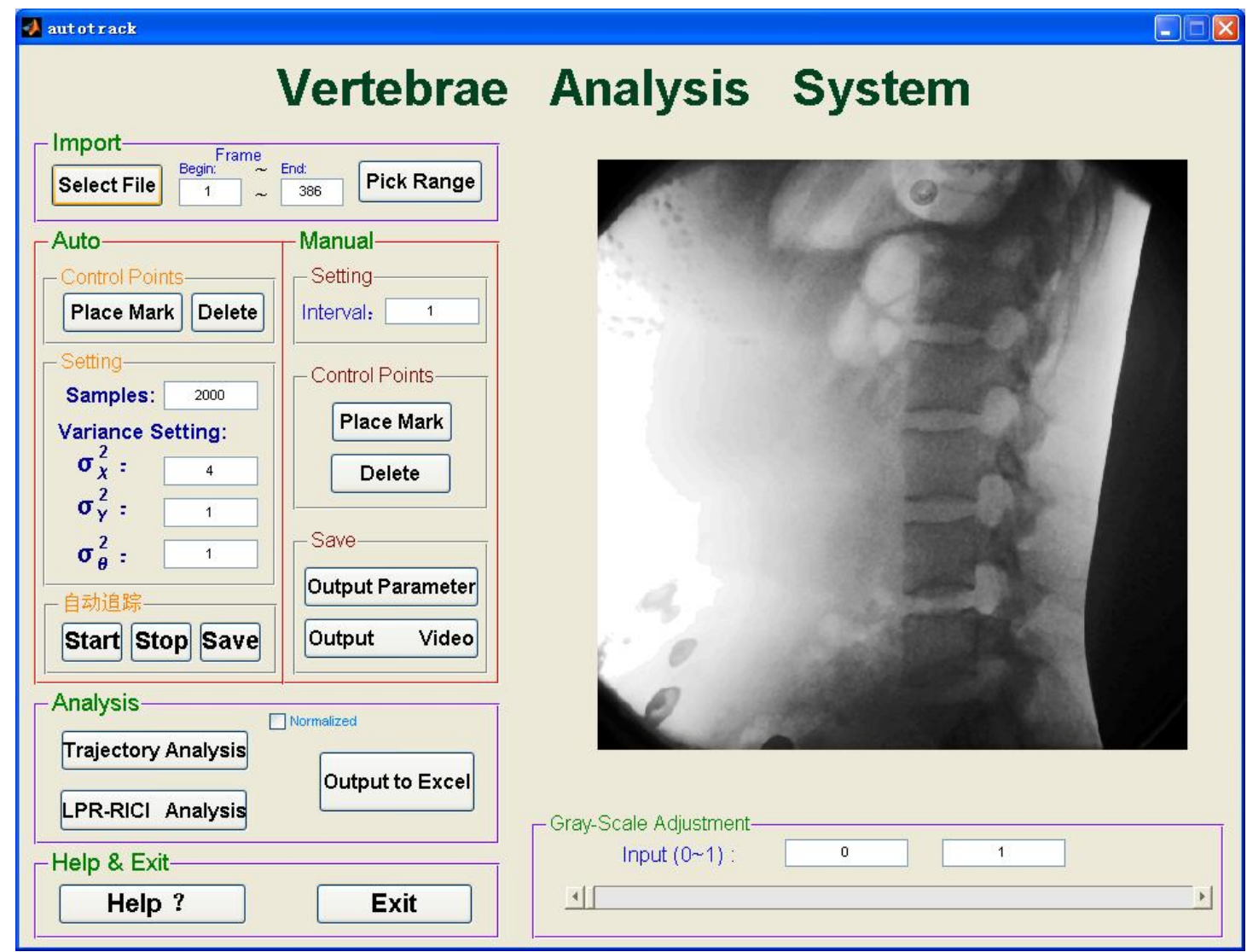


Figure 3

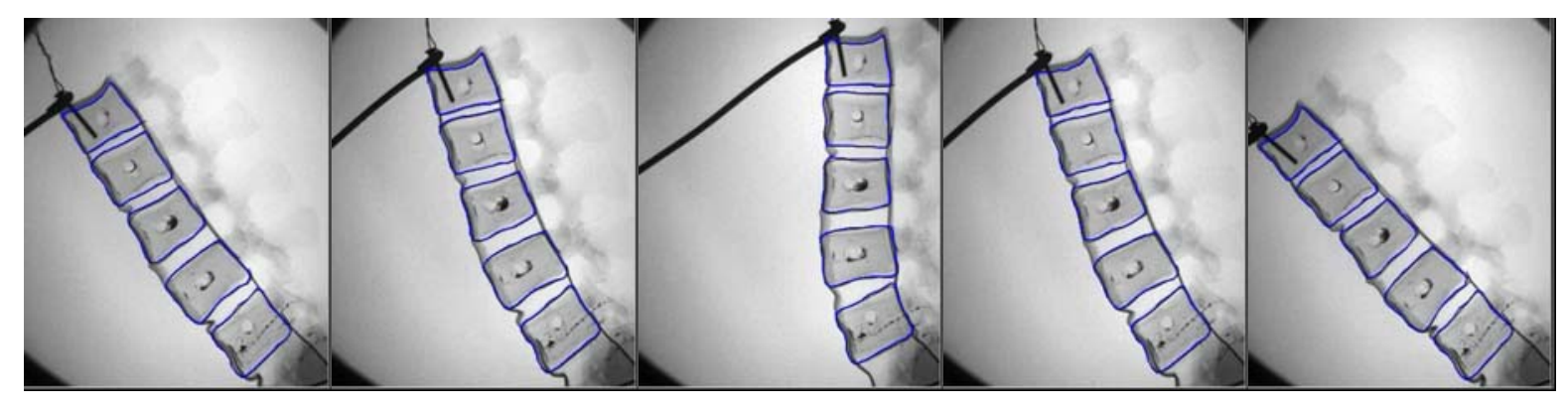

$\begin{array}{lllll}\text { (a) Frame: } 1 & \text { (b) Frame: } 50 & \text { (c) Frame: } 100 & \text { (d) Frame: } 150 & \text { (e) Frame: } 200\end{array}$ 
Figure 4
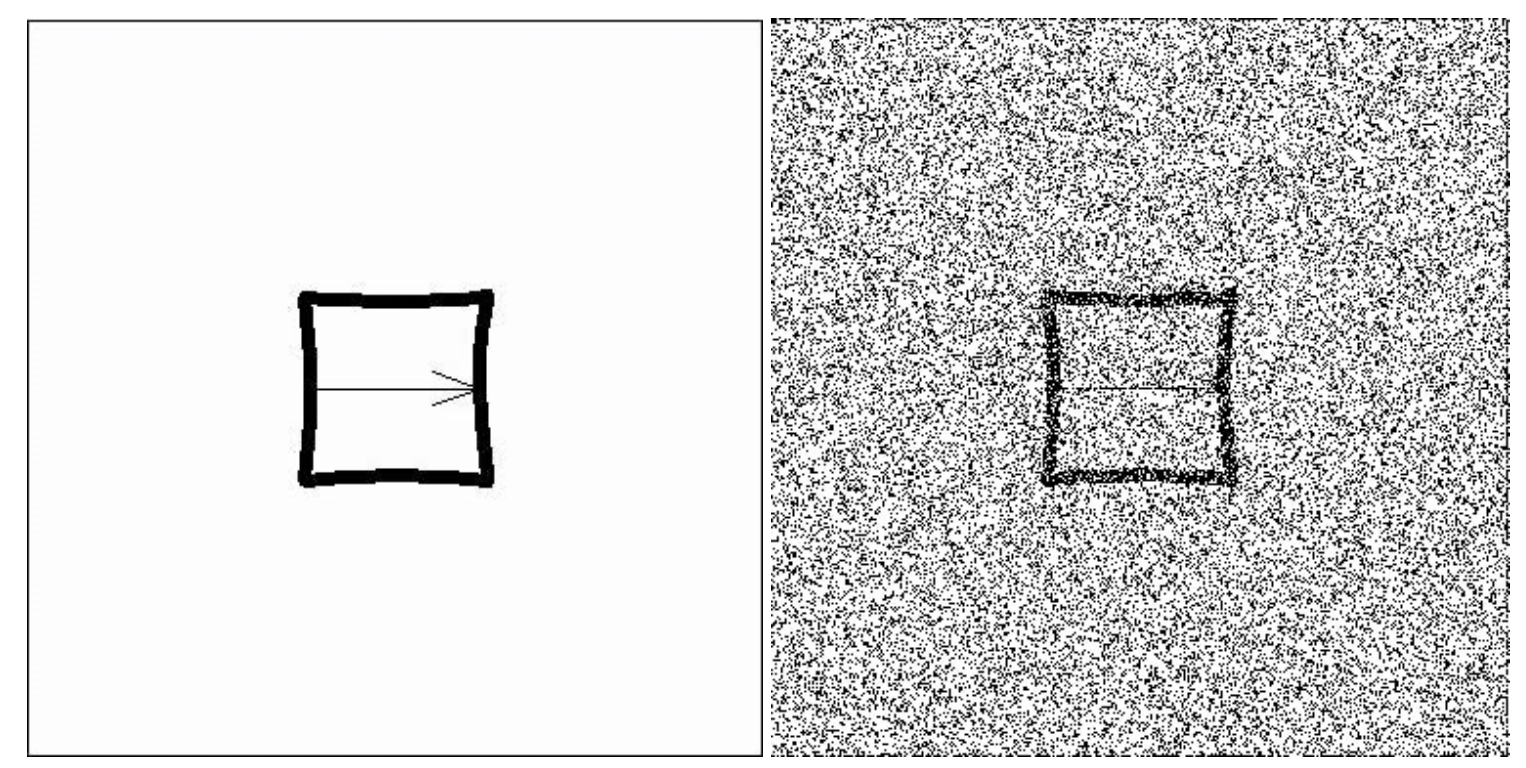

(a)

(b) 
Figure 5

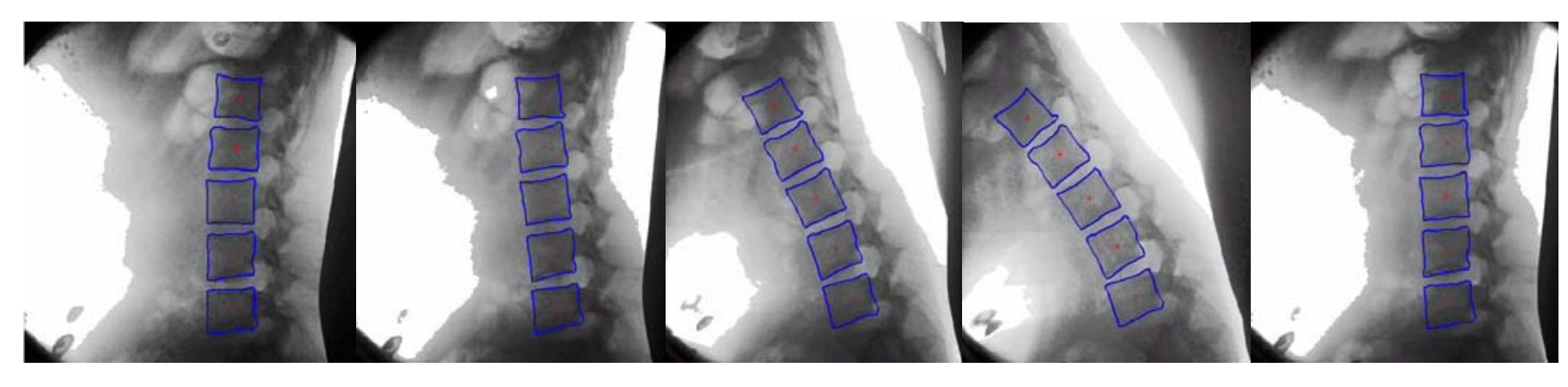
(a) Frame: 1
(b) Frame: 50
(c) Frame: 100
(d) Frame: 150
(e) Frame: 200 
Figure 6
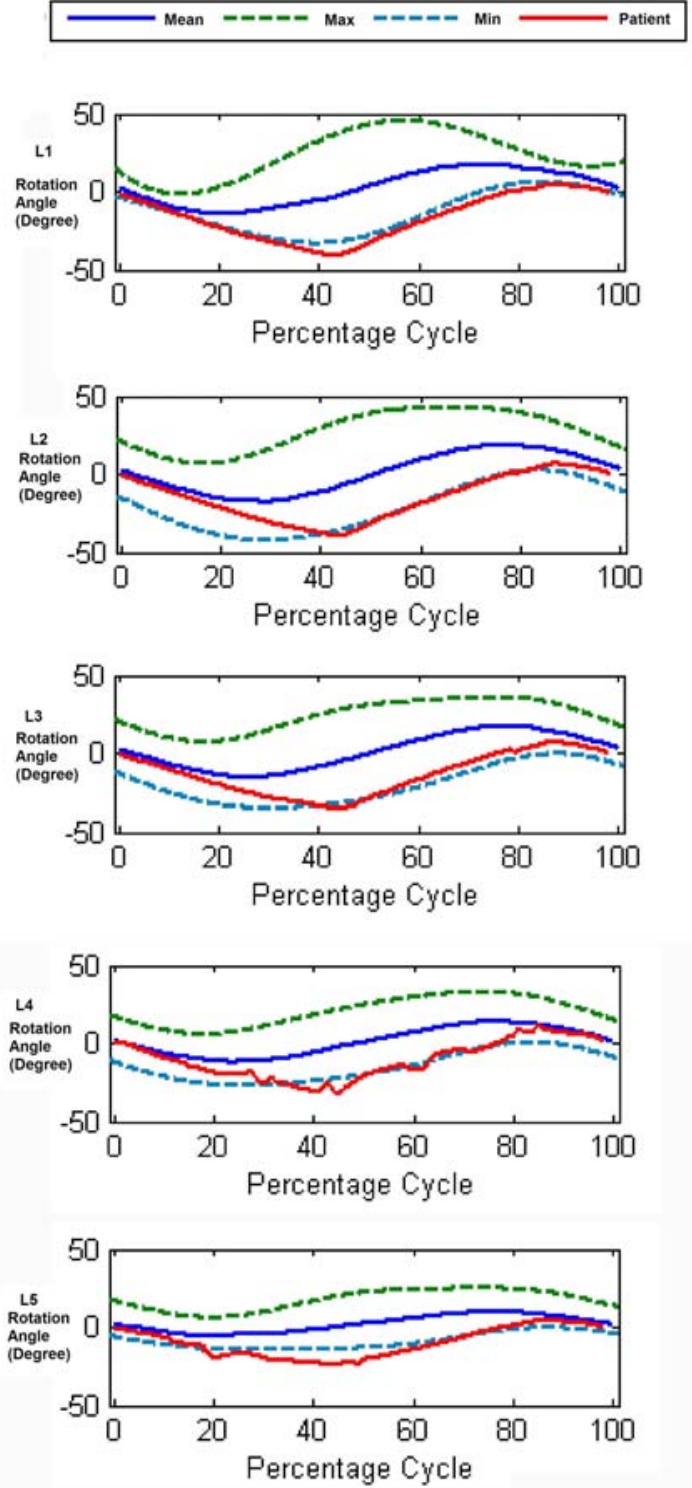

(a)
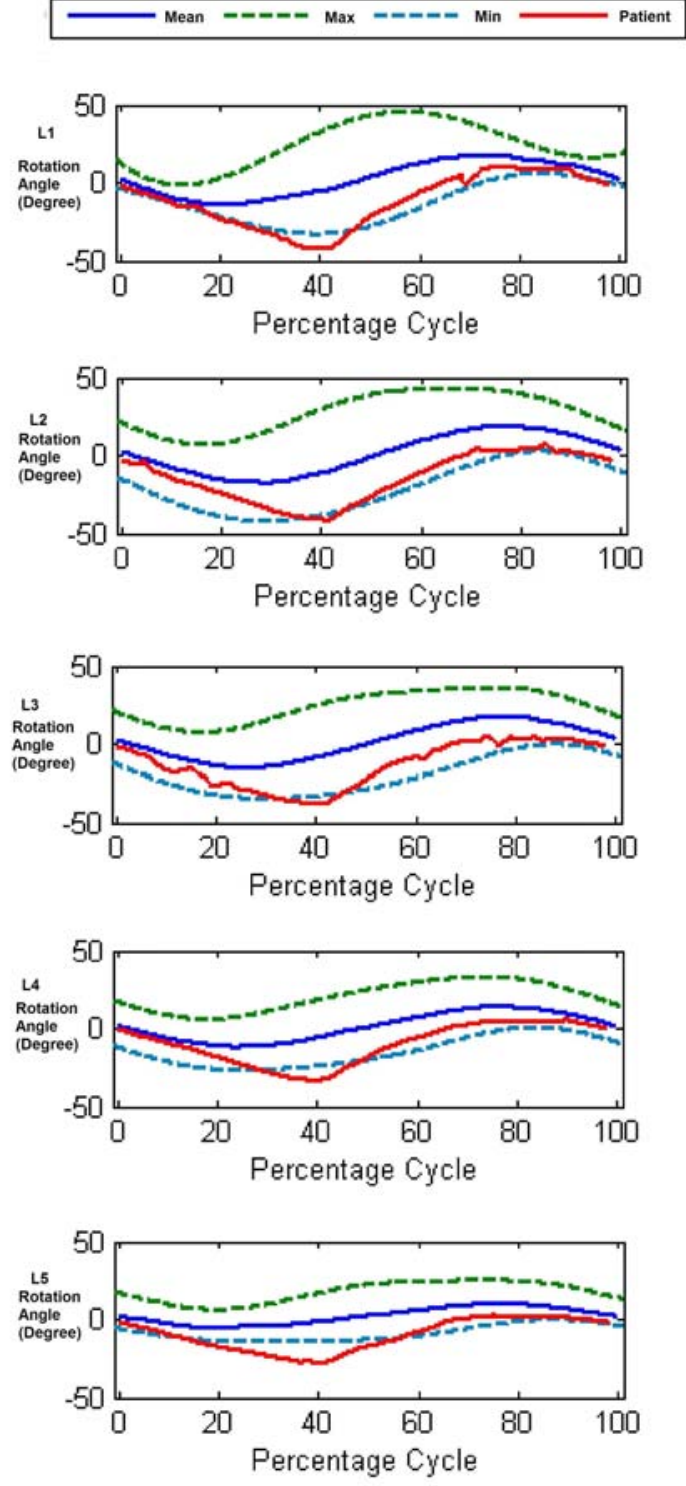

(b) 
Figure 7

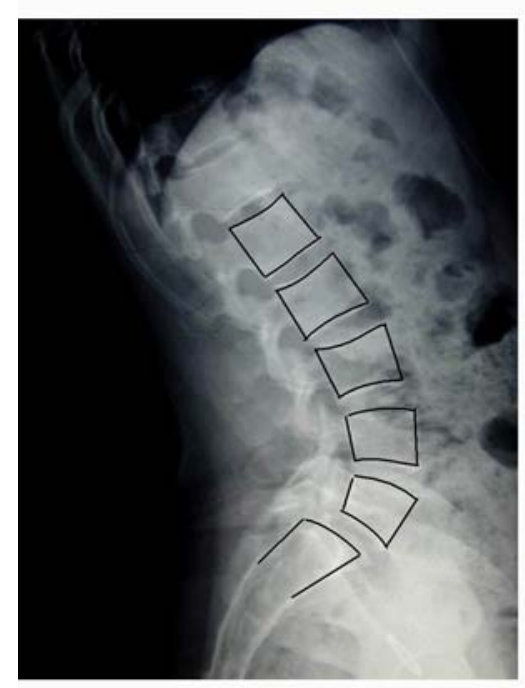

(a)

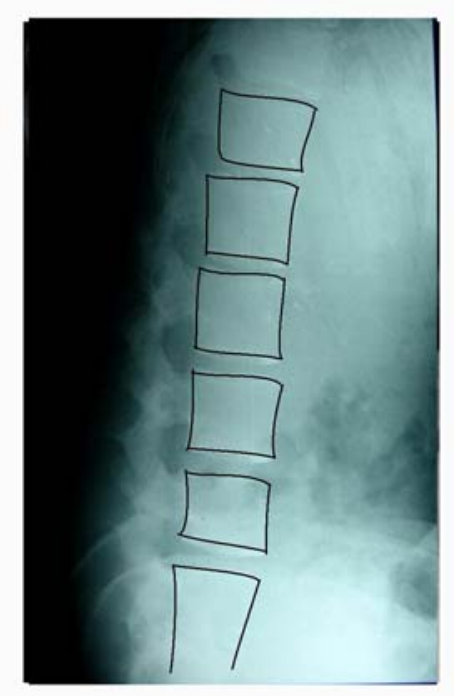

(d)

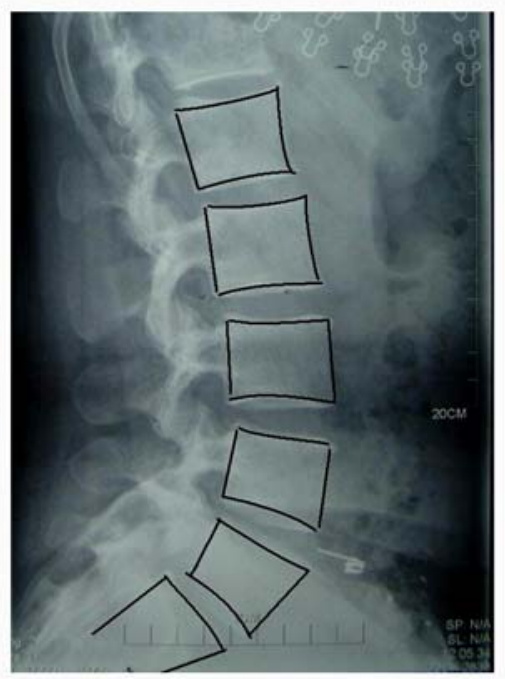

(b)

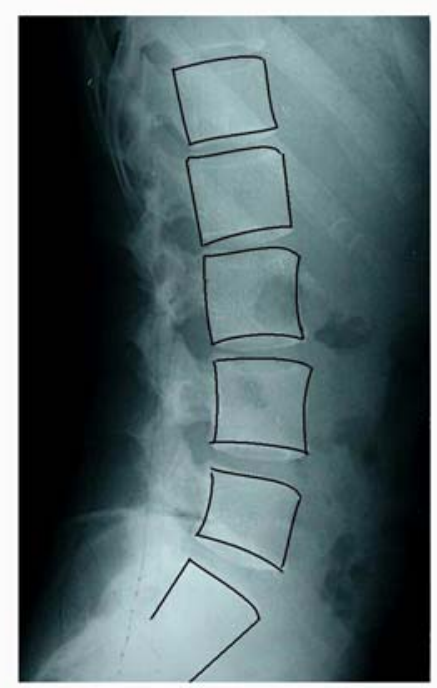

(e)

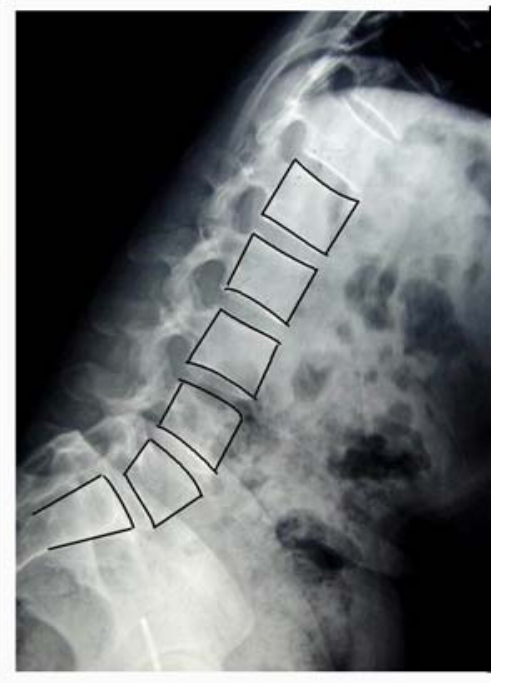

(c)

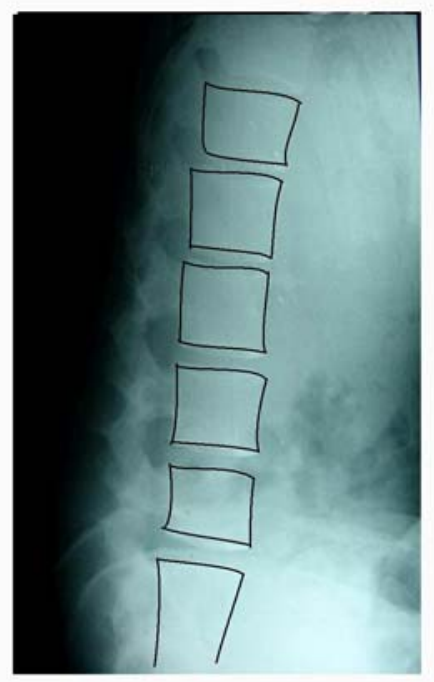

(f) 